\title{
A Practical Approach to Numerically Predicting a Maneuvering Vessel in Waves Oriented to Maritime Simulator
}

\author{
Shengke Ni $\mathbb{D}^{1}{ }^{1}$ Zhengjiang Liu, $^{1}$ Yao Cai, ${ }^{1}$ and Teng Zhang ${ }^{2}$ \\ ${ }^{1}$ Navigation College, Dalian Maritime University, Dalian 116026, China \\ ${ }^{2}$ School of Naval Architecture Engineering, Dalian University of Technology, Dalian 116024, China \\ Correspondence should be addressed to Shengke Ni; 15841101155@163.com
}

Received 21 August 2020; Revised 5 October 2020; Accepted 14 October 2020; Published 26 October 2020

Academic Editor: Taoreed Owolabi

Copyright (c) 2020 Shengke Ni et al. This is an open access article distributed under the Creative Commons Attribution License, which permits unrestricted use, distribution, and reproduction in any medium, provided the original work is properly cited.

To improve the behavioral realism of maritime simulator, the mathematical model of ship heave and pitch motion in regular waves is proposed. To avoid the influence of irregular frequency by two-dimensional Frank source and sink distribution method, the multiparameter conformal mapping method was adopted to solve the hydrodynamic problem of ship's transverse sections, and then the integration of the hydrodynamic coefficients and the wave exciting forces for the whole ship hull was obtained using the Salvesen-Tuck-Faltinsen (STF) strip method. The Abkowitz model was used for the horizontal maneuverability motion equations, and the ship heave and pitch motion mathematical model in regular waves can be built considering the maneuvering factors. To verify the credibility and applicability of the improved model, the Mariner vessel with a Froude number of 0.2 was taken as the simulation case; when Mariner vessel sails in head waves, the maximum relative error between the calculated results and the experimental results is $23.6 \%$ for amplitude response operators of ship heave and pitch motion, and the calculated results and the experimental results have the same trend. When turning maneuvers in regular waves are carried out, the time history of ship pitch and pitch motions in regular waves does not show the sinusoidal periodic change any more, but the time history of heave and pitch motion still possesses periodic change to some extent, at which the period is about the time required for the turning motion to finish a circle. When turning maneuvers are carried out for 3000 seconds, the time required for computer operation is about $487.6 \mathrm{~s}$, and the real-time requirements of maritime simulator are satisfied. Thus, the mathematical model of ship heave and pitch motion established in this paper can be effectively applied to the maritime simulator.

\section{Introduction}

As an efficient and economical virtual simulation training platform, maritime simulator illustrated in Figure 1 has been widely used in navigation training, maritime analysis, and port evaluation, and the ship motion mathematical model is the key to improving behavioral realism for maritime simulator by Jin and Yin [1]. At present, most of the ship motion mathematical models for maritime simulator are generated using commercial software, which is quite expensive and difficult to update, and greatly affects the sustainable development of maritime simulator. Therefore, it is urgent to establish ship motion mathematical model with high accuracy and applicability.

The hydrodynamic coefficients and the wave exciting force coefficients of the ship can be obtained by empirical formula, and the mathematical model of ship heave and pitch motion in regular waves was built by Li and Yang [2]. By supposing the ship as "box ship," the wave forces calculation model was established for dynamic positioning simulator. The empirical formula and the "box ship" assumption reduce the accuracy of ship motion mathematical model, which limits the scope of application of ship type in Qian et al. [3].

With the rapid improvement of computer computing and storage capacity, the numerical prediction of ship motion based on two-dimensional (2D) and three-dimensional (3D) theory has been greatly developed. In the field of two-dimensional theory, Hou [4] adopted the ordinary strip method to predict ship heave and pitch motions, and numerical results of the S175 container ship can be obtained 


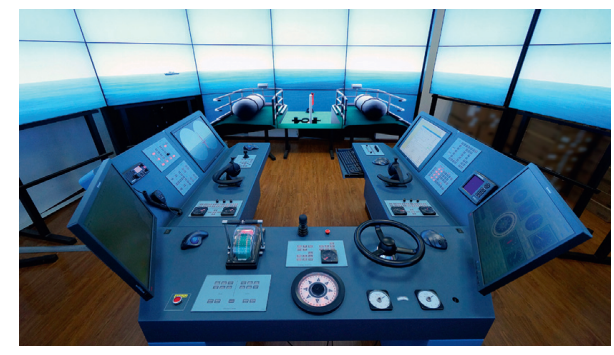

Figure 1: Schematic diagram of maritime simulator.

and analyzed under different wavelengths and wave heights. The ordinary strip method is only applicable to the head sea condition, which greatly limits the application scope. The STF strip method was presented to predict ship motions in regular waves by Salvesen et al. [5], and the comparisons between numerical results and the experimental results show good agreement for most cases. Although the hydrodynamic coefficients of transverse sections were calculated by Frank source and sink distribution method, they were easy to be affected by irregular frequency, which leads to numerical divergence. Guevel and Bougis [6] solved the radiation-diffraction problem of a ship sailing in waves via the threedimensional frequency domain Green function method, and the numerical analysis of the mixed distribution method and the source distribution method was carried out. The geometric characteristics of the hull surface and flow field could be taken into account by the three-dimensional frequency domain method. Newman [7] found that the calculation of the three-dimensional frequency Green function with forward speed is quite complex and time-consuming, and the accuracy of the numerical prediction for ship motions was quite poor especially when the ship speed was high. In the three-dimensional frequency domain. Nakos [8] carried out the numerical prediction of ship heave and pitch motion responses via high-order Rankine source method in the paper [9]. In the time domain, Chen et al. [10] adopted three-dimensional Rankine source function to obtain the hydrodynamic problems and motions of ship in regular waves. However, radiation condition is hard to achieve in short wavelengths for Rankine source method. Sun et al. [11] established the hydrodynamic analysis model of the ship in still water by the three-dimensional transient free-surface Green function. Clement [12] discovered that the three-dimensional transient free-surface Green function can be a solution to an ordinary differential equation. And Li et al. [13] solved three-dimensional transient free-surface Green function by modified precise integration method. Sun and Ren [14] further extended the three-dimensional transient freesurface Green function to diffraction problem and carried out numerical predictions of ship motions in regular waves. The three-dimensional transient free-surface Green function was not stable for the evaluation the hydrodynamic forces of the ship with flare, which reduced the scope of application of the ship type. Datta et al. [15] carried out modifications to the ship hull which is non-wall side, so that the three-dimensional transient free-surface Green function can be applied to solve the hydrodynamic problem without numerical divergence.
However, the ship needs maneuvering in waves to achieve the scheduled navigation status; the influence of maneuver factors on ship motions in regular waves should be considered effectively. Yasukawa $[16,17]$ conducted the turning test, stopping test, and zigzag test in regular waves for SR108 container ship model. The free-running model maneuvering test could obtain reliable results, but experimental methods are not easy to be carried out. Based on the two-dimensional Frank source sink distribution method, the wave forces and hydrodynamic forces acting on the ship were calculated and directly added to the ship maneuvering motion equations in still water, and maneuvering motion and swaying motion of ship in regular waves can be predicted by Zhu et al. [18]. The waves forces acting on the ship were computed on the basis of "box ship" assumption and were added to the maneuvering mathematical group (MMG) equations, where the mathematical model of ship motion with six degrees of freedom in waves was established for the maritime simulator by Zhang and Yin [19]. The interaction among ship, propeller, and rudder was not well considered in the model of MMG. Based on the high-order Rankine source method, Zhang et al. [20] established the boundary integral equation of disturbance velocity potential considering the horizontal maneuvering motion, and the coupled equations of ship maneuvering motion and swaying motion were solved using the two-time-scale approach. Wang et al. [21] carried out the CFD (computational fluid dynamics) simulations of free-running ship under different operational conditions. Although the complex characteristics of flow field were considered by CFD-based method, the computational effort required could still be enormous. Several hundreds of CPU (Central Processing Unit) hours can be taken to simulate simply several seconds maneuver, which is difficult to meet the real-time simulation requirements of maritime simulator.

To verify the proposed ship motion mathematical model for maritime simulator, within two-dimensional strip theory, the multiparameter conformal mapping method is employed to solve the hydrodynamic forces and wave exciting forces acting on the ship's transverse sections, and the influence of wave direction angle and wave length on ship heave and pitch motion is studied. The Abkowitz model is employed to predict the ship heave and pitch motion in waves under maneuvering conditions, and the effects of rudder angle on heave and pitch motion are studied. The ship heave and pitch motion model can be integrated into maritime simulator by Visual Studio software, where the visualization of ship motion mathematical model can be achieved. Mariner vessel and YUKUN vessel are taken as study cases, and comparisons are presented between calculated results and experimental data in the paper [5]. Thus, the ship motion model proposed for maritime simulator in this paper is validated.

\section{Mathematical Formulations}

2.1. Coordinate Systems. As shown in Figure 2, there are two coordinates systems used for solving the ship hydrodynamic and motion problems; the space-fixed coordinate system 


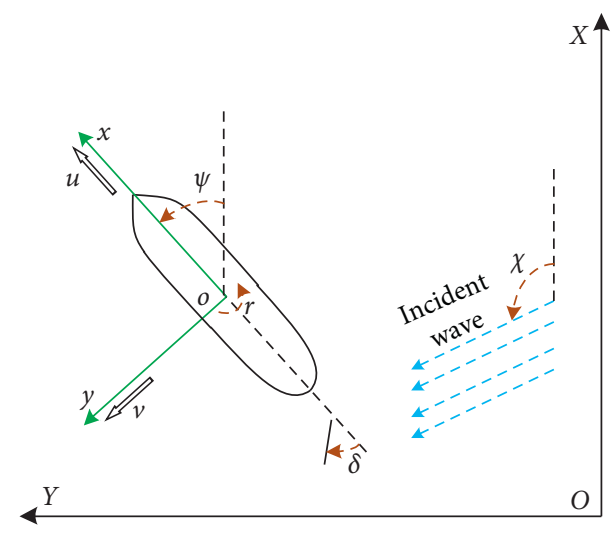

Figure 2: Definition of coordinate systems.

$O X Y Z$ is adopted to keep track of position and Euler angles of the ship, $O X Y$ plane describes the mean free surface with $o z$-axis pointing upward. The body-fixed coordinate system $O X Y Z$ is coincident with space-fixed coordinate system $O X Y Z$ at time $t=0$. The $o x$-axis points toward the bow, the $o y$-axis points toward the port side, and the origin $o$ is fixed at the midship. In the body-fixed coordinate system $O X Y Z$, the $u$ and $v$ are translational velocities, and $r$ is rotational rate. $\psi$ is heading angle of the bow. $\delta$ is rudder angle. $\chi$ is wave angle of incident wave, and $\chi=0^{\circ}$ represents the wave angle propagating positively along the $o x$-axis.

The relationship of the abovementioned two coordinate systems at time $t$ should be

$$
\left\{\begin{array}{l}
X=x \cos (\psi)-y \sin (\psi)+x_{o} \\
Y=x \sin (\psi)+y \cos (\psi)+y_{o} \\
Z=z
\end{array}\right.
$$

where $\left(x_{o}, y_{o}, 0\right)$ are the coordinates of origin $o$ in the spacefixed coordinate system.

In the space-fixed coordinate system, the wave elevation $\zeta_{I}(t)$ at origin $o$ is given in the indefinite water depth as [22]

$$
\zeta_{1}(t)=\zeta_{0} \cos \left[k\left(x_{o} \cos \chi+y_{o} \sin \chi\right)-\omega t\right],
$$

where $\zeta_{0}$ is the amplitude of incident wave; $\omega$ is the absolute frequency of incident wave, $k=\left(\omega^{2} / g\right)$ denotes wave number; and $g$ denotes gravitational acceleration.

In the body-fixed coordinate system, the incident wave potential $\phi_{I}(x, y, z, t)$ can be

$$
\phi_{I}(x, y, z, t)=\frac{\zeta_{0} g}{\omega} e^{k z} \sin [k \omega-\omega t],
$$

where $\omega=x \cos (\chi-\psi)+y \sin (\chi-\psi)+x_{o} \cos \chi+y_{o} \sin \chi$.

2.2. Establishment of Abkowitz Model. The Abkowitz model describes horizontal maneuvering motion, which includes surge, sway, and yaw motions [23]. In the body-fixed coordinate, the dynamic equations of horizontal maneuvering motion can be given as

$$
\left\{\begin{array}{l}
\left(m-X_{\dot{u}}\right) \dot{u}=f_{1}(u, v, r, \delta, W), \\
\left(m-Y_{\dot{v}}\right) \dot{v}+\left(m x_{G}-Y_{\dot{r}}\right) \dot{r}=f_{2}(u, v, r, \delta, W), \\
\left(m x_{G}-N_{\dot{v}}\right) \dot{v}+\left(I_{z z}-N_{\dot{r}}\right) \dot{r}=f_{3}(u, v, r, \delta, W),
\end{array}\right.
$$

where $m$ denotes mass of ship; $x_{G}$ denotes the $x$-coordinate value of the ship's center of gravity; $I_{z z}$ denotes the moment of inertia about $z$-axis; $X_{\dot{u}}, Y_{\dot{v}}$, and $Y_{\dot{r}}$ denote added mass on the $o x$-axis and $o y$-axis; $N_{\dot{v}}$ and $N_{\dot{r}}$ denote add moment of inertia about the $o z$-axis; $f_{1}, f_{2}$, and $f_{3}$ are nonlinear force functions with respect to $u, v, r, \delta$, and $W ; u$ denotes surge velocity; $v$ denotes sway velocity; $r$ denotes yaw velocity; $\delta$ denotes rudder angle; and $W$ is corresponding to secondorder wave force.

Under several assumptions, $f_{1}, f_{2}$, and $f_{3}$ can be given as shown by Bai [24]. In order to solve equation (4), hydrodynamic coefficients need to be nondimensionalized by Fossen [23], and the differential equation of horizontal maneuvering motion can be solved accurately by the fourthorder Runge-Kutta method.

\subsection{Multiparameter Conformal Mapping Transformation of} Ship's Transverse Sections. Figure 3 is a diagram of conformal transformation between the plane $x^{\prime} o^{\prime} y^{\prime}$ and the plane $\xi^{\prime} o^{\prime} \eta^{\prime}$; the $o^{\prime} x^{\prime}$-axis points downward; $o^{\prime} y^{\prime}$-axis points starboard; $y_{w}$ denotes half-width of transverse section; $D$ denotes the draft of transverse section; $C_{0}$ denotes contour line of transverse section; $\mathbf{n}$ denotes the unit normal vector pointing into the transverse section; $\vec{n}$ denotes the unit normal vector of a quarter unit circle; $\gamma$ denotes radian angle of a quarter unit circle; and $R$ denotes the radius of unit circle.

The outer part of the ship's transverse section contour $C_{0}$ in the $z^{\prime}$ plane is transformed into the outer part of the unit circle in the $\varsigma^{\prime}$ plane by conformal mapping transformation:

$$
z^{\prime}=\sum_{s=1}^{M-1} a_{2 s-1}\left(\varsigma^{\prime}\right)^{-2 s+1}
$$

where $z^{\prime}=x^{\prime}+i y^{\prime} ; \varsigma^{\prime}=\xi^{\prime}+i \eta^{\prime}=R \cdot e^{i \gamma} ; 0 \leq \gamma \leq(\pi / 2) ; a_{2 s-1}$ is transformation parameters; and $M$ is the number of conformal mapping transformation parameters.

Let

$$
w^{\prime}=\frac{z^{\prime}}{\varsigma^{\prime}}=p^{\prime}+i q^{\prime}
$$

Complex number $z_{i}^{\prime}$ denotes the $i$ th transformation point on the contour line $C_{0}$, and complex number $w_{i}^{\prime}$ is $i$ th complex number $w^{\prime}$ and can be defined as

$$
w_{i}^{\prime}=\frac{z_{i}^{\prime}}{\varsigma_{i}^{\prime}}=p_{i}+\mathrm{i} q_{i}, \quad i=1,2,3, \ldots, M-1, M,
$$

where $\varsigma_{i}^{\prime}=e^{i \gamma_{i}}, \gamma_{i}=((i-1) \pi /[2(M-1)])$ is the $i$ th radian angle in the first quadrant of the unit circle corresponding to the $i$ th transformation point $z_{i}^{\prime}$, and $p_{i}$ and $q_{i}$ are the real part and the imaginary part of the complex number $w_{i}^{\prime}$, respectively. 


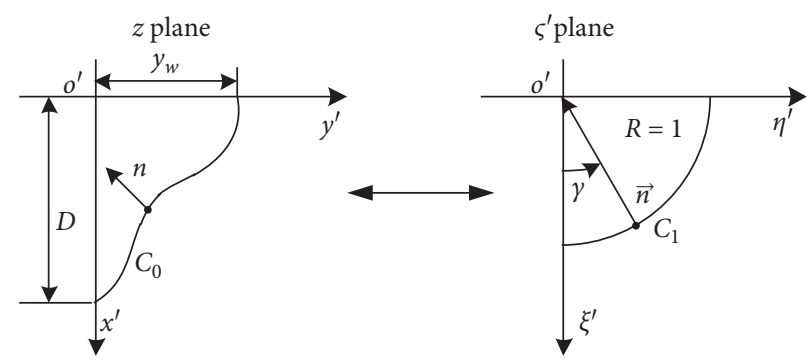

FIGURE 3: Conformal mapping transformation between half transverse section and a quarter unit circle.

In combination with equations (5)-(7), the equation can be given as

$$
p_{i}=a_{-1}+a_{1} \cos \left(2 \gamma_{i}\right)+\cdots+a_{2 M-3} \cos \left[(2 M-2) \gamma_{i}\right]
$$

If equation (8) is solved, then $M$ conformal transformation coefficients can be deduced as

$$
\left\{\begin{array}{l}
a_{-1}=\frac{1}{2(M-1)}\left(p_{1}+2 \sum_{i=2}^{M-1} p_{i}+p_{M}\right), \\
a_{2 l-1}=\frac{1}{M-1}\left[p_{1}+\sum_{i=2}^{M-1} p_{i} \cos \left(\frac{i-1}{M-1} l \pi\right)+(-1)^{f} p_{M}\right], \quad l=1,2, \ldots, M-2, \\
a_{2 M-1}=\frac{1}{2(M-1)}\left[p_{1}+2 \sum_{i=2}^{M-1}(-1)^{i} p_{i}+(-1)^{M-1} p_{M}\right] .
\end{array}\right.
$$

To obtain the conformal transformation coefficients, it is necessary to give the initial value of the transformation point $z_{i}$ as

$$
z_{i}^{\prime}=a_{-1} \varsigma_{i}^{\prime}+\frac{a_{1}}{\varsigma_{i}^{\prime}}
$$

where $a_{-1}=\left(D+y_{w}\right)$ and $a_{1}=\left(\left(D-y_{w}\right) / 2\right)$.

Figure 4 shows the calculation flow of $M$ conformal mapping coefficients. Since the initial $M$ transformation points are not on the data line of the ship's transverse section, the conformal mapping coefficients can be solved iteratively by Westlake and Wilson [25].

In Figure 4, $\sigma$ is the designated error tolerance, which can be set according to the ship shape, scale, and other parameters.

\subsection{Establishment of Ship Heave and Pitch Motion Equations} in Regular Waves. The transverse section is assumed to carry out harmonic heave motion in the plane $x^{\prime} o^{\prime} y^{\prime}$ with an oscillation frequency $\omega$ and a small displacement amplitude $h_{0}$ :

$$
x^{\prime}=h_{0} \cos \left(\omega t+\varepsilon_{0}\right),
$$

where $\varepsilon_{0}$ is the phase angle.
Within the potential flow theory, radiation velocity potential $\phi_{R}$ in the $\varsigma^{\prime}$ plane can be transformed from $z^{\prime}$ plane, and the following requirement should be satisfied with respect to the parameters $R$ and $\gamma$ [26]:

(1) The Laplace equation:

$$
\left(\frac{\partial^{2}}{\partial R^{2}}+\frac{1}{R} \frac{\partial}{\partial R}+\frac{\partial^{2}}{\partial \gamma^{2}}\right) \phi_{R}=0
$$

(2) Symmetrical equation about $o^{\prime} x^{\prime}$-axis:

$$
\frac{\partial \phi_{R}}{\partial \gamma}=0, \quad \gamma=0, R>1
$$

(3) The linearized free-surface condition in infinite depth:

$$
k \sum_{i=0}^{M-1}(-1)^{i}(2 i-1) a_{2 i-1} R^{-(2 i-1)} \cdot \phi_{R}+\frac{\partial \phi_{R}}{\partial \gamma}=0, \quad \gamma=\frac{\pi}{2}, R>1 .
$$

(4) The boundary condition on the surface of transverse section:

$$
\frac{\partial \phi_{R}}{\partial R}=\dot{x}^{\prime} \frac{\partial x^{\prime}}{\partial R}
$$




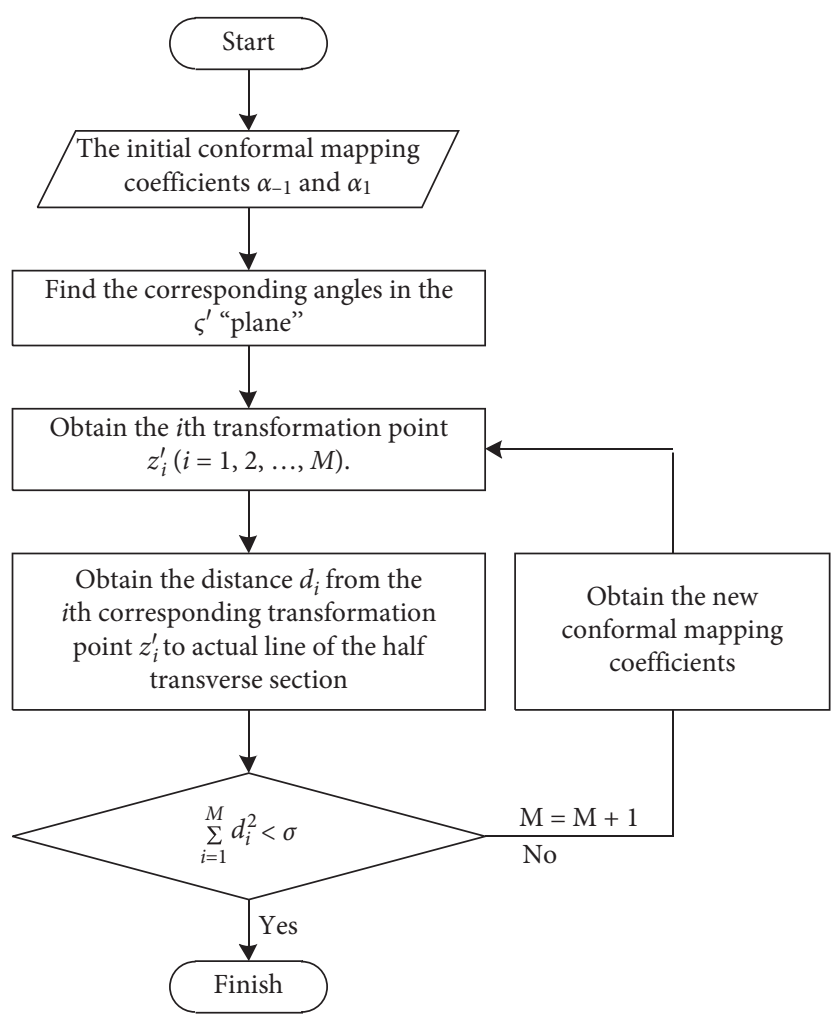

FIGURE 4: The flowchart of conformal mapping.

(5) The radiation condition at infinity:

$$
\phi_{R} \longrightarrow 0, \quad R \longrightarrow \infty \text { on } z=0 .
$$

In combination with equations (12)-(16), the radiation velocity potential $\phi_{R}$ can be obtained [27].

From Bernoulli's equation, the hydrodynamic force $f_{R}$ on the transverse section of unit length in the $o^{\prime} x^{\prime}$-direction is obtained as

$$
f_{R}=-\rho \int_{C_{0}} \frac{\partial \phi_{R}}{\partial t} \mathrm{~d} y^{\prime}
$$

The hydrodynamic force $f_{R}$ can also be expressed as

$$
f_{R}=-m^{\prime} \frac{\mathrm{d} \dot{x}^{\prime}}{\mathrm{d} t}-N^{\prime} \dot{x}^{\prime}
$$

In combination with equations (17) and (18), the hydrodynamic coefficients $m^{\prime}$ and $N^{\prime}$ can be solved.

When ship advances with a constant forward speed $U$ in regular waves, the linear differential equations of coupled heave and pitch motion can be given as

$$
\left\{\begin{array}{c}
\left(m+A_{33}\right) \ddot{z}+B_{33} \dot{z}+C_{33} z+A_{35} \ddot{\theta}+B_{35} \dot{\theta}+C_{35} \theta=F_{z} \cos \left(\omega_{e} t+\varepsilon_{z}\right) \\
\left(J_{y y}+A_{55}\right) \ddot{\theta}+B_{55} \dot{\theta}+C_{55} \theta+A_{53} \ddot{z}+B_{53} \dot{z}+C_{53} z=F_{\theta} \cos \left(\omega_{e} t+\varepsilon_{\theta}\right)
\end{array}\right.
$$

where $\theta$ is the pitch angle; $J_{y y}$ is the moment of inertia about $y$-axis; $\omega_{e}$ is the wave encounter frequency; $\varepsilon_{z}$ is the phase angle of heave wave exciting force; $\varepsilon_{\theta}$ is the phase angle of pitch wave exciting force moment; $A_{33}, B_{33}, A_{35}$, $B_{35}, A_{55}, B_{55}, A_{53}$, and $B_{53}$ are the hydrodynamic coefficients; $F_{z}$ and $F_{\theta}$ are the heave wave force amplitude and pitch wave moment amplitude, respectively, induced by incident wave; $C_{53}, C_{55}, C_{33}$, and $C_{35}$ are the hydrostatic restoring coefficients; for better consideration of speed and end of ship effects, based on STF strip method [5], the abovementioned coefficients can be obtained by integrating the hydrodynamic coefficients $m^{\prime}$ and $N^{\prime}$ of transverse section.

To eliminate numerical divergence induced by the initial disturbance of the fluid, the right terms of equation (19) need to be multiplied by the ramp function $f_{m}$ [28], where $f_{m}$ can be set as

$$
f_{m}= \begin{cases}\frac{1}{2}\left[1-\cos \left(\frac{\pi t}{T_{w}}\right)\right], & t<T_{w}, \\ 1, & t \geq T_{w},\end{cases}
$$


where $T_{w}$ can be set as two times of the incident wave period.

When the initial time $t=0$, the displacement and velocity value of the ship heave and pitch motion are set as $z=0$, $\dot{z}=0, \theta=0$ and $\dot{\theta}$; the time history of the ship heave and pitch motion can be obtained by solving equation (19) with the fourth-order Runge-Kutta method [4].

When the ship advances in regular waves with a constant forward speed, the ship heave and pitch motion can be directly solved by equation (19).

When the ship advances in regular waves under maneuvering conditions, equations (4) and (19) need to be coupled and solved to obtain the ship heave and pitch motion results.

Figure 5 shows the flowchart of numerical prediction of ship heave and pitch motion ( $\Delta t$ is time step).

\section{Numerical Simulation and Result Analysis}

3.1. Study Cases. Mariner vessel and YUKUN vessel are adopted as the study cases in this paper, and parameters of the ships are shown in Table $1 . L, B$, and $D$ denote length of ship, breadth of ship, and draft of ship, respectively; $k_{y y}$ denotes radius of pitch inertia moment; $\nabla$ denotes volume of displacement; $C_{b}$ denotes block coefficient; $A_{r}$ denotes area of rudder; and $D_{0}$ denotes diameter of propeller.

\subsection{Conformal Mapping of the Transverse Section for Mariner} Vessel. The Mariner vessel is divided into twenty-one transverse sections. The transverse sections are numbered from the stern to bow. No. 0 is for the first section numbered, No. 1 is the second transverse section, and so on. As shown in Figure 6, "Lewis mapping" denotes mapping of transverse section by two conformal mapping coefficients $\left(a_{-1}\right.$ and $\left.a_{1}\right)$, "Current mapping" denotes mapping of transverse section by multiparameter conformal mapping coefficients $(M>2)$, and "Actual transverse section" denotes data points obtained from the actual transverse section contour line. The designated error tolerance $\sigma$ is set as $B / 40$ for "Current mapping." The number of mapping coefficients for No. 4 and No. 5 transverse sections are 17 and 19, respectively, by "Current mapping."

As seen from Figure 6, for No. 4 and No. 5 of the ship's transverse section, the data points of actual ship's transverse section are all located on the lines generated by "Current mapping," while only a few of the data points on of actual ship's transverse section are located on the lines generated by the "Lewis mapping" [29]. Therefore, the actual ship's transverse sections can be better fit with multiparameter coefficient conformal method, and an effective solver can be provided for ship hydrodynamic solution.

3.3. Numerical Simulation and Analysis of the Motions of Mariner Vessel in Regular Waves. To verify the reliability and accuracy of the mathematical model of ship motion in waves established in this paper, the numerical simulation of the motion for Mariner vessel in regular waves is carried out. Mariner vessel advances at constant speed Froude number $\mathrm{Fn}=0.2(\mathrm{Fn}=(U / \sqrt{g L}))$.
As shown in Figures 7 and $8, z_{0}$ denotes amplitude of heave motion, $\left(z_{0} / \zeta_{0}\right)$ denotes response amplitude operator of heave motion, $\theta_{0}$ denotes amplitude of heave motion, $\left(\theta_{0} L / 2 \zeta_{0}\right)$ denotes response amplitude operator of heave motion, and $(\lambda / L)$ denotes wavelength to ship length ratio. "Current method" denotes numerical results obtained by the presented method in this paper; "OSM method" denotes numerical results obtained by OSM (ordinary strip method); "Salvesen method" denotes numerical results obtained by Salvesen et al. [5], where the hydrodynamic coefficients are solved by Frank source and sink method; and "Experiment" denotes experimental results given by Salvesen et al. [5].

In Figures 7 and 8, for the response amplitude operator (RAO) of the heave motion of Mariner vessel, when the length to wavelength ratio $(\lambda / L)$ is in the range of $0.8 \sim 2.1$, the numerical results of "Current method"; both "Salvesen method" and "OSM method" are in good agreement with the results of "Experiment." Relative error between "Current method" and "Experiment" is within 8.8\%, the relative error between the "Salvesen method" and "Experiment" is within $9.1 \%$, and the relative error between "OSM method" and "Experiment" is within 9.7\%. For the response amplitude operator of the pitch motion of the Mariner vessel, when the length to wavelength ratio $(\lambda / L)$ is in the range of $0.8 \sim 2.1$, the relative error between "Current method" and "Experiment" is within $12.9 \%$, relative error between the "Salvesen method" and "Experiment" is within 3.5\%, and relative error between "OSM method" and "Experiment" is within 14.6\%. The numerical results of "Current method" are in better agreement with the "Experiment" results than "OSM method," especially in the peak area of pitch response amplitude operator curve; "OSM method" results are lower than other methods.

As the wave angle $\chi$ is in the range of $0^{\circ} \sim 360^{\circ}$ and the amplitude of wave $\zeta_{0}$ is $1 \mathrm{~m}$, Figures 9 and 10 show the amplitude of heave and pitch motion under different $(\lambda / L)$.

In Figure 9, the amplitude of heave motion is symmetrical with respect to the wave angle $\chi=180^{\circ}$. With the increase of $(\lambda / L)$, the fluctuation range of the amplitude of heave motion varying with the wave angle is narrowed, and the amplitude of heave motion gradually tends to $1 \mathrm{~m}$.

In Figure 10, the amplitude of the ship's pitch motion is symmetrical with respect to the wave angle $\chi=180^{\circ}$, and the wave angle $\chi=90^{\circ}$ is located at the trough of the pitch motion amplitude curve; due to the asymmetry of the hull geometry around midship, the amplitude of pitch motion is not 0 , but the value is about $0.02^{\circ}$.

For Figures 11-16, the parameters of incident wave are set as follows: when $(\lambda / L)=1.0$, the amplitude of incident wave is $\zeta_{0}=1 \mathrm{~m}$, the wave angle is $\chi=135^{\circ}$ at $t=0$; the rudder angles of Mariner vessel are $\delta=0^{\circ}, \delta=15^{\circ}$, and $\delta=25^{\circ}$, respectively.

As shown in Figures 11-16, when the rudder angle $\delta=0^{\circ}$ is applied to the Mariner vessel, the ship advances forward straightly with constant speed in regular waves, and the time histories of the heave and pitch motion change sinusoidal periodically. When a nonzero rudder angle is applied to Mariner vessel, the ship carries out turning motions in regular waves, the wave encounter frequency for Mariner 


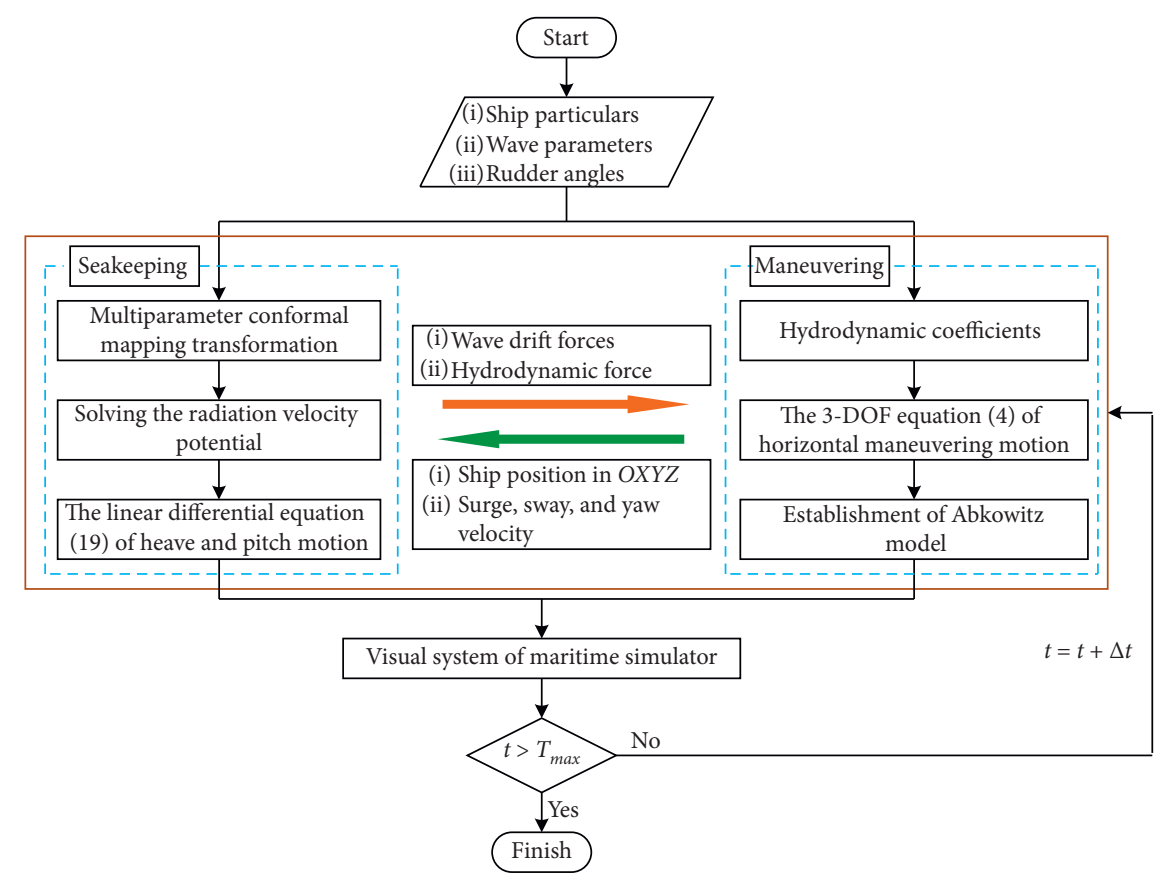

Figure 5: The flowchart for numerical prediction of ship heave and pitch motion.

TABLE 1: Main particulars of the Mariner vessel and YUKUN vessel.

\begin{tabular}{lcccccccc}
\hline Ship & $L(\mathrm{~m})$ & $B(\mathrm{~m})$ & $D(\mathrm{~m})$ & $k_{y y}$ & $\nabla\left(\mathrm{m}^{3}\right)$ & $C_{b}$ & $A_{r}\left(\mathrm{~m}^{2}\right)$ & $D_{0}(\mathrm{~m})$ \\
\hline Mariner & 160.93 & 23.17 & 8.23 & $0.25 \mathrm{~L}$ & 18541 & 0.61 & 30.01 & 6.71 \\
YUKUN & 105 & 18 & 5.4 & $0.25 \mathrm{~L}$ & 5710.2 & 0.56 & 11.8 & 3.8 \\
\hline
\end{tabular}

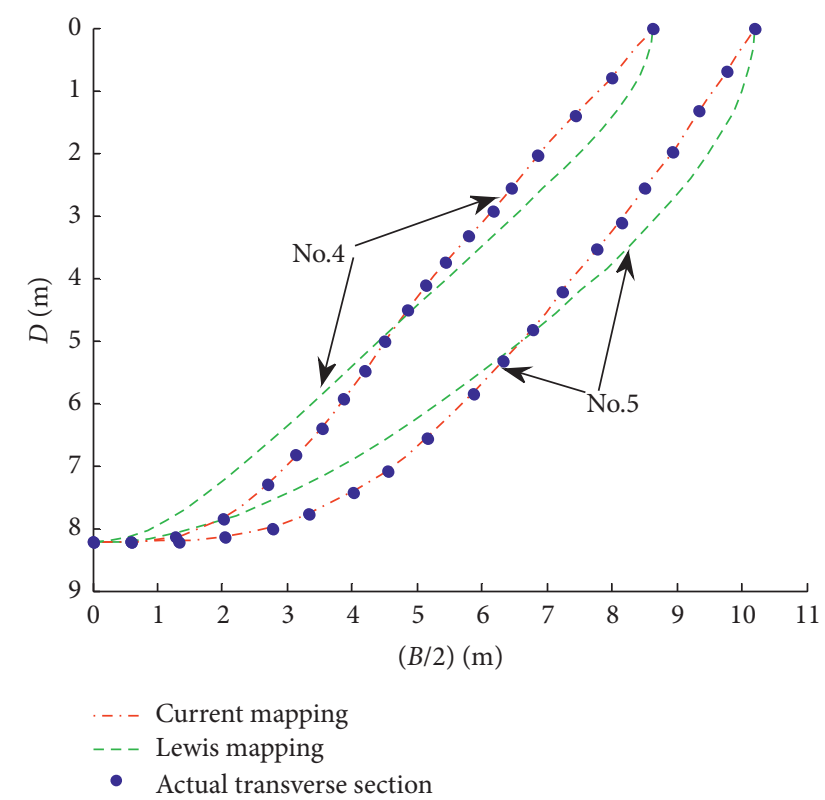

FIGURE 6: Results of conformal mapping for transverse section No. 4 and No. 5.

vessel changes all the time, time history of the ship's heave and pitch motion is not sinusoidal and still possesses periodicity to some extent, and its period is about the time needed for the Mariner vessel to finish a turning circle.

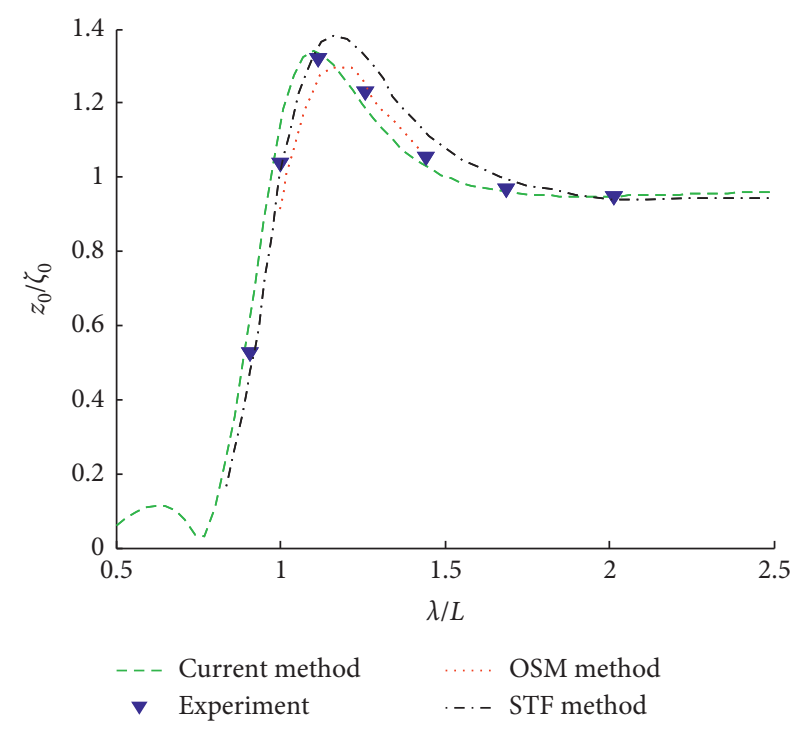

FIGURE 7: Heave response amplitude operators of Mariner vessel in head waves.

HpZ440 workstation is used as the simulation platform in this paper. The hardware parameters are Intel ${ }^{\circledR} \mathrm{Xeon}^{\mathrm{TM}} \mathrm{e} 5$ $1650 v 3$ CPU @ $3.5 \mathrm{GHz}$, memory RAM is $32.00 \mathrm{~GB}$. To verify the real-time performance of the proposed mathematical model of ship motion, the ship pitch and heave motion under a certain rudder angle for Mariner vessel are 


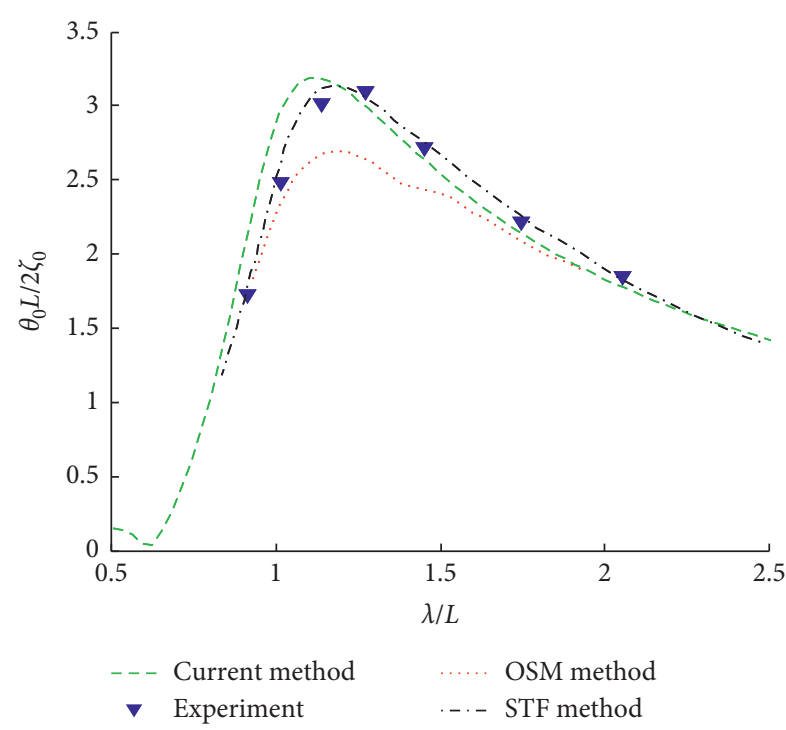

FIGURE 8: Pitch response amplitude operators of Mariner vessel in head waves.

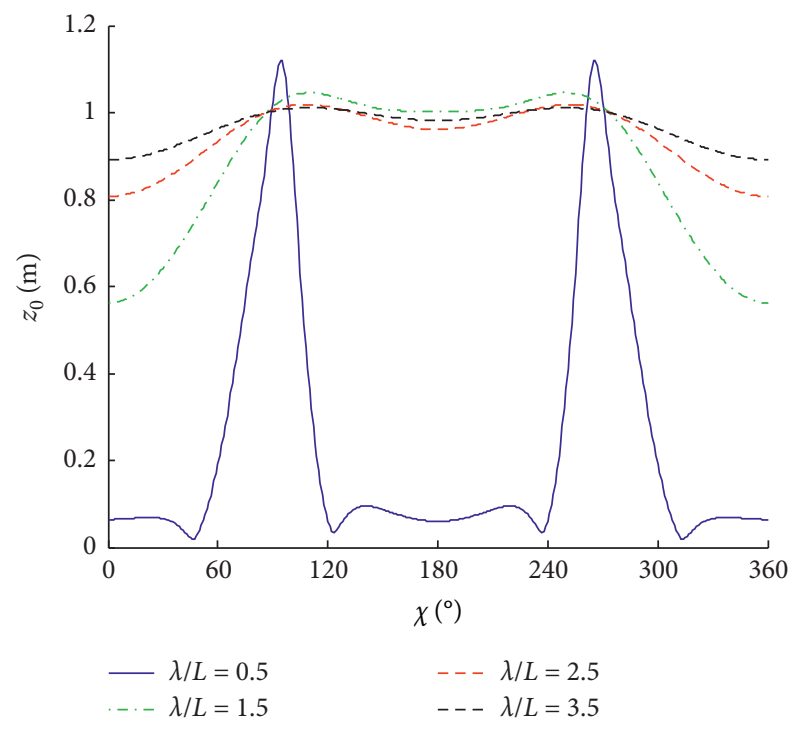

Figure 9: Amplitude of heave motion for Mariner vessel under different $(\lambda / L)$.

taken as an example; when the Mariner ship carried out motion for $3000 \mathrm{~s}$, the time consuming of CPU is about $487.6 \mathrm{~s}$, which is much lower than the actual time of the ship motion, thus providing a solid guarantee for the real-time performance of the maritime simulator.

3.4. Visualization of the Motions for the YUKUN Vessel in Regular Waves. Based on the Visual Studio software, the numerical simulation program of the mathematical model of the ship heave and pitch motion in regular waves is developed, which is connected to the simulation platform of the maritime simulator to realize the real-time solution and visualization of the ship heave and pitch motion model.



FIgURE 10: Amplitude of pitch motion for Mariner vessel under different $(\lambda / L)$.

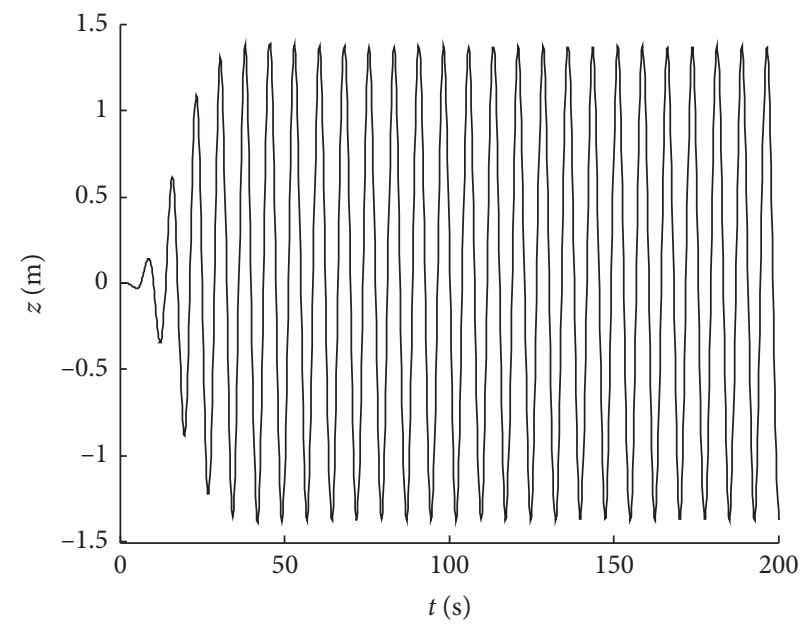

FIGURE 11: The time history of heave motion for Mariner vessel $\left(\delta=0^{\circ}\right)$

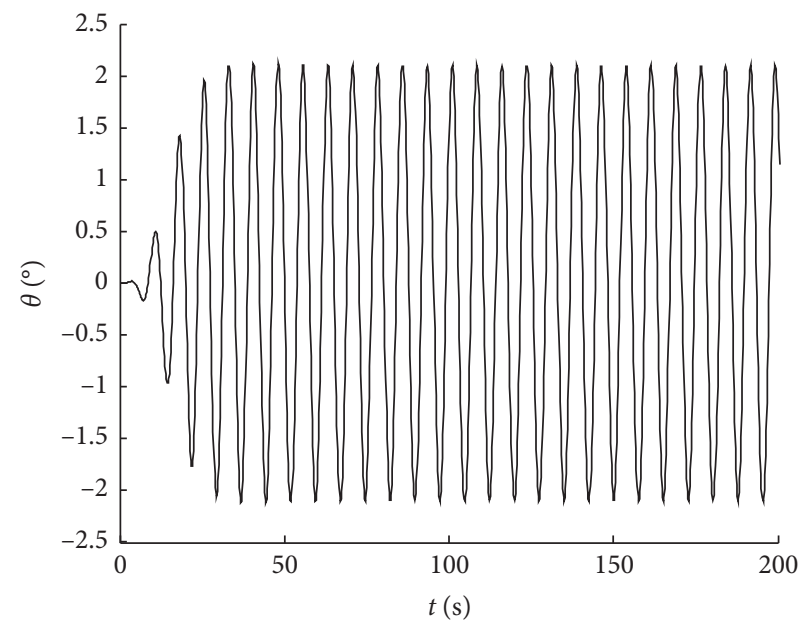

FIgURE 12: The time history of pitch motion for Mariner vessel $\left(\delta=0^{\circ}\right)$. 


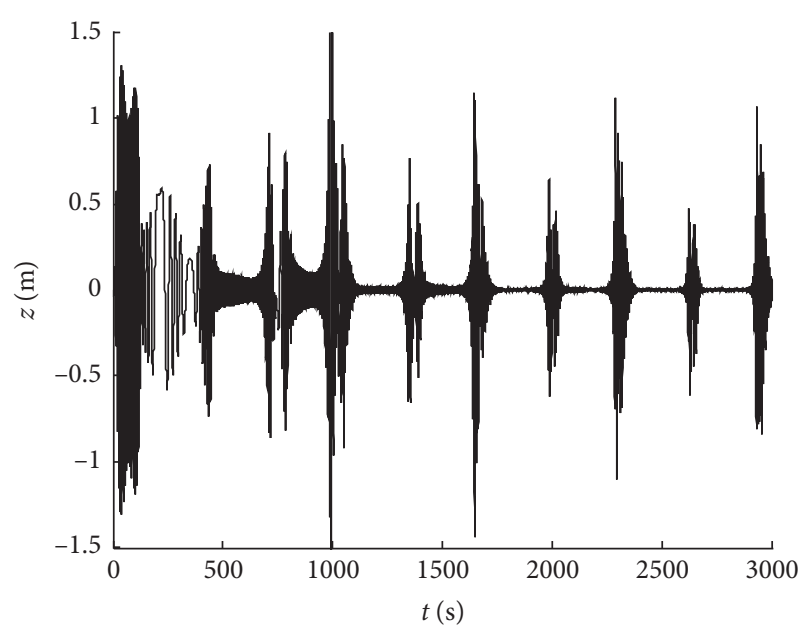

FIgURE 13: The time history of heave motion for Mariner vessel $\left(\delta=15^{\circ}\right)$.

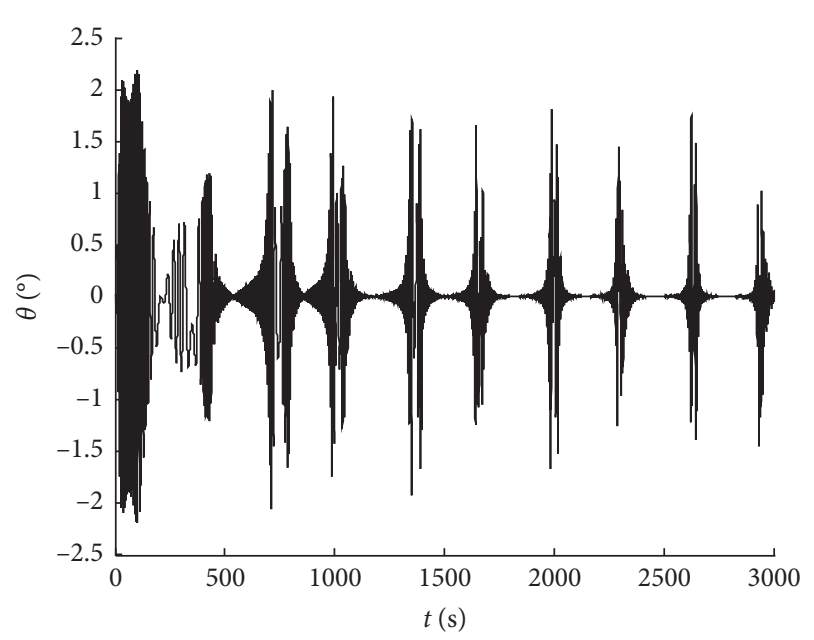

FIGURE 14: The time history of pitch motion for Mariner vessel $\left(\delta=15^{\circ}\right)$.

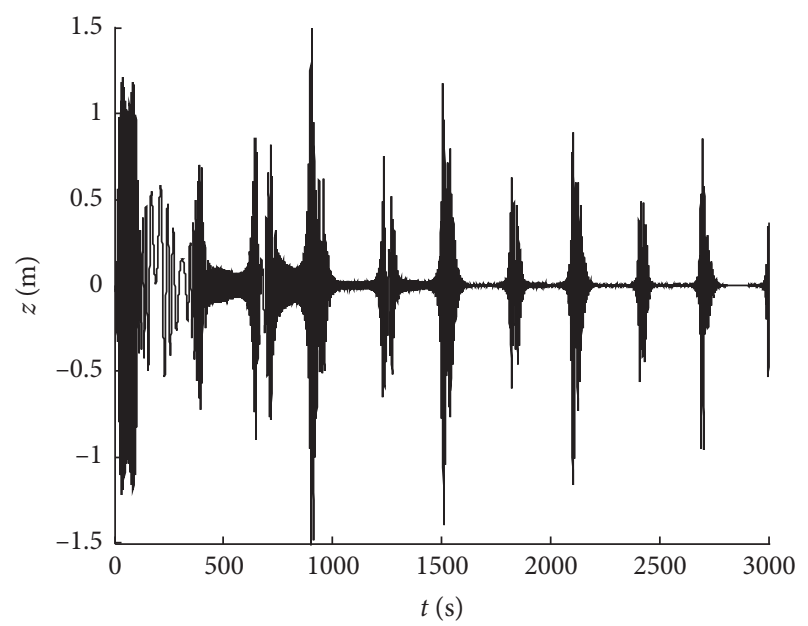

FIGURE 15: The time history of heave motion for Mariner vessel $\left(\delta=25^{\circ}\right)$.

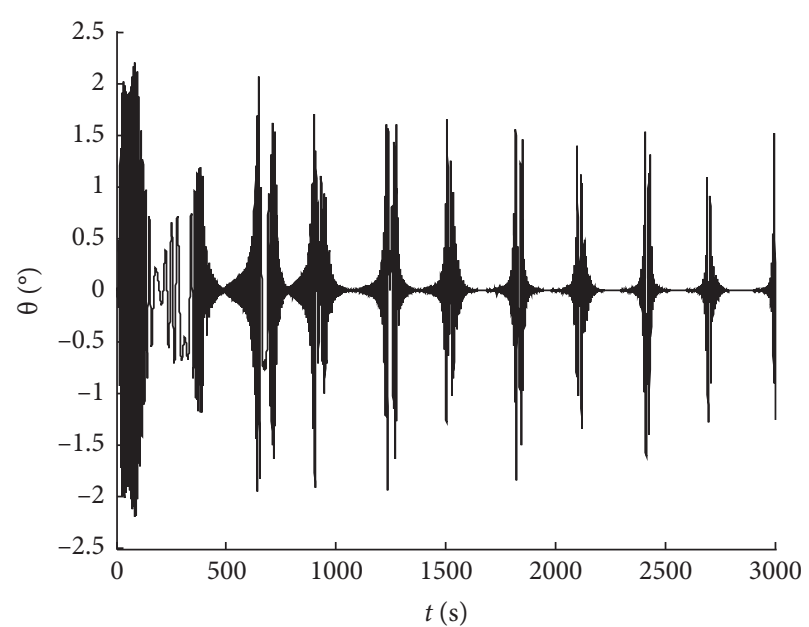

Figure 16: The time history of pitch motion for Mariner vessel $\left(\delta=25^{\circ}\right)$.

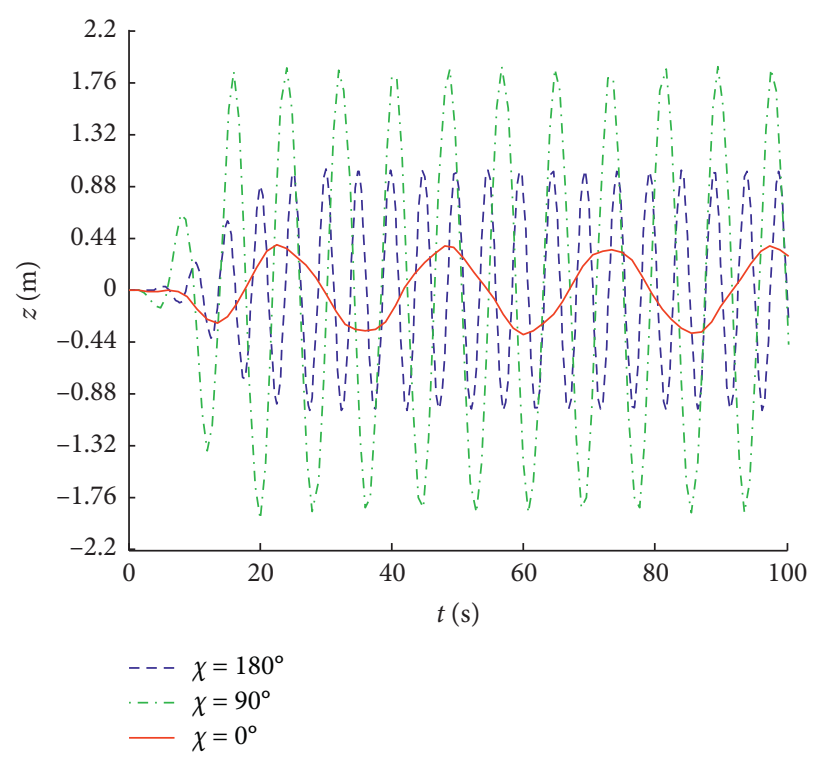

FIGURE 17: The time history of heave motion for YUKUN vessel $\left(\chi=0^{\circ}, \chi=90^{\circ}\right.$, and $\left.\chi=180^{\circ}\right)$.

YUKUN vessel advances straightly at the design speed of $16.7 \mathrm{kn}(1 \mathrm{kn}=0.514 \mathrm{~m} / \mathrm{s})$ in regular waves. The parameters of incident waves are set as follows: the amplitude of wave is $\zeta_{0}=2 \mathrm{~m} ;(\lambda / L)$ is 1 ; the wave angles are $\chi=0^{\circ}, \chi=90^{\circ}$, and $\chi=180^{\circ}$, respectively.

From Figures 17 and 18, when YUKUN ship advances straightly with constant speed in regular waves, under the action of different wave angles, the ship heave and pitch motion change sinusoidal period, and the numerical value does not diverge. It proves the robustness and applicability of the program developed in this paper, which can further model the motion of other ship types for the maritime simulator.

Under the above wave parameters and working conditions, when the wave angle is $\chi=180^{\circ}$, Figure 19 is the visual schematic diagram of the motion attitude of the YUKUN vessel on the simulation platform of the maritime simulator. 


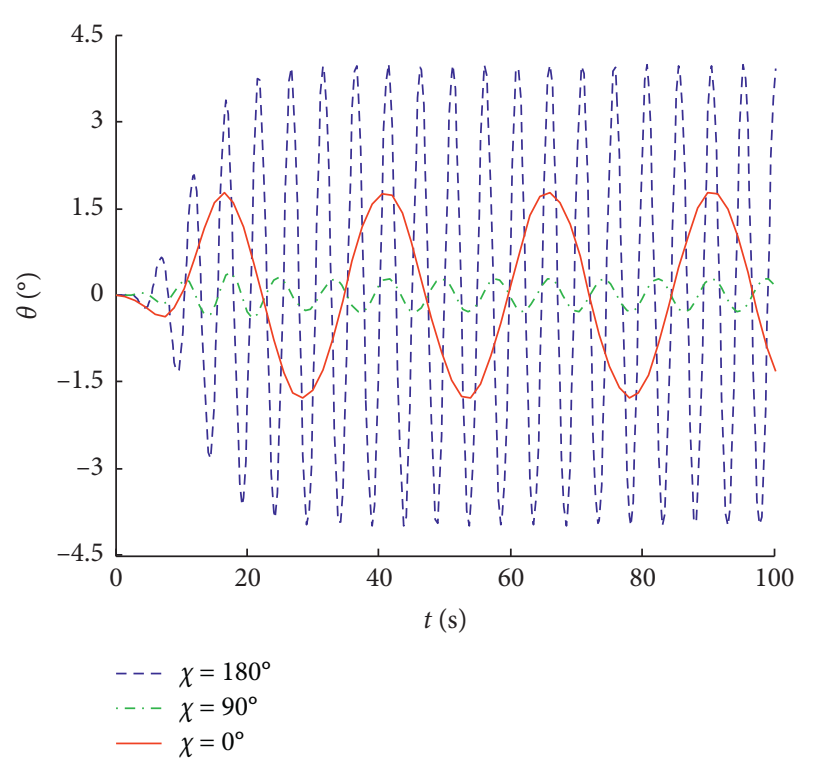

FIgURE 18: The time history of pitch motion for YUKUN vessel $\left(\chi=0^{\circ}, \chi=90^{\circ}\right.$, and $\left.\chi=180^{\circ}\right)$.

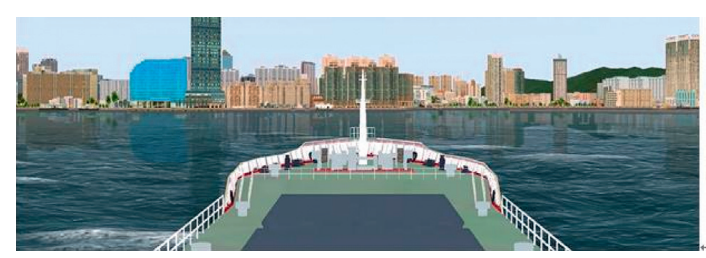

Figure 19: The heave displacement of ship is $1.01 \mathrm{~m}$ and pitch displacement of ship is $-1.96^{\circ}$, respectively, when $t$ is $44.7 \mathrm{~s}$.

From Figure 19, the ship motion mathematical model built in this paper could be applied to the simulation platform of maritime simulator.

\section{Conclusions}

(1) In this paper, aiming at the problem that the empirical formula and three-dimensional theory are not suitable for ship motion modeling of maritime simulator, within two-dimensional strip theory, a new mathematical model of the ship heave and pitch motion in regular waves via multiparameter conformal mapping method has been established.

(2) To avoid the influence of the irregular frequency inherent in the two-dimensional Frank source and sink distribution method, this paper makes a highprecision fitting of the ship's transverse section based on the multiparameter conformal mapping method, so as to provide a reliable solver for the study of ship's hydrodynamic force and motion in waves within the two-dimensional strip theory.

(3) Based on the numerical calculation of Mariner vessel's motion, the effects of different wavelength, wave angle, and rudder angle on the ship heave and pitch motion are analyzed. When Froude number of ship is set as 0.2 , the relative error between the response amplitude operator of heave motion and the experimental value is $8.8 \%$, which shows good agreement with the experimental results when Mariner vessel advances straightly in regular waves at a constant speed; under a certain rudder angle, the period of time history of the heave and pitch motion is about the time required for the ship to finish a circle in regular waves, and the consuming time of $\mathrm{CPU}$ is far less than the actual operation time of the ship, which effectively guarantees the real-time performance of the maritime simulator.

(4) The time history of heave and pitch motion for YUKUN vessel under various wave angles is calculated, and the stable and convergent numerical results are obtained. Based on Visual Studio, the simulation platform of maritime simulator is connected to realize the visualization of ship's motion attitude. Thus, the mathematical model of ship heave and pitch motion proposed in present paper has achieved engineering application.

(5) Based on the theory of linear potential flow, this paper studies the ship heave and pitch motion in regular waves. In fact, when a ship sails in the ocean, the rolling motion of the ship is also present due to either direct wave forcing or interaction with other ship motions. Furthermore, during a turning circle due to the development of a rolling angle during this maneuver, especially if the ship's speed during the maneuver is high and for special ship types (e.g., RO$\mathrm{RO}$ ferries), this angle might be large enough to have a significant impact on ship motion. Because the viscous forces account for a large part, the theory of linear potential flow is difficult to meet the modeling requirements. It is necessary to incorporate more freedom for establishing mathematical model of ship motion under complex sea conditions and operational conditions.

\section{Data Availability}

The data used to support the findings of this study are available from the corresponding author upon request.

\section{Conflicts of Interest}

The authors declare that they have no conflicts of interest regarding the publication of this paper.

\section{Acknowledgments}

This work was supported in part by the National Natural Science Foundation of China (Grant no. 51309041), the National Natural Science Foundation of China (Grant no. 51909022), the Natural Science Foundation of Liaoning Province (Grant no. 2019-BS-024), and the Fundamental Research Funds for the Central Universities (Grant nos. 3132019136 and 3132019347). 


\section{References}

[1] Y. C. Jin and Y. Yin, "Maritime simulators: convention and technology," Navigation of China, vol. 33, no. 1, pp. 1-6, 2010.

[2] Z. F. Li and Y. S. Yang, "Simulation of heaving and pitching of ship in regular wave," Journal of Dalian Maritime University, vol. 28, no. 4, pp. 13-16, 2002.

[3] X. B. Qian, Y. Yin, X. F. Zhang, and Y. Li, "Influence of irregular disturbance of sea wave on ship motions," Journal of Traffic and Transportation Engineering, vol. 7, pp. 116-124, 2016.

[4] S. X. Hou, "Simulation on ship heave and pitch motions in head sea," Master thesis, Dalian Maritime University, Dalian, China, 2015.

[5] N. Salvesen, E. O. Tuck, and O. Faltinsen, "Ship motions and sea loads," Transactions Society of Naval Architects and Marine Engineers, vol. 78, pp. 250-287, 1970.

[6] P. Guevel and J. Bougis, "Ship-motions with forward speed in infinite depth," International Shipbuilding Progress, vol. 29, no. 332, pp. 103-117, 1982.

[7] J. N. Newman, The Approximation of Free-Surface Green Functions, Cambridge University Press, Cambridge, UK, 1992.

[8] D. E. Nakos, Ship wave patterns and motions by a three dimensional Rankine panel method, Ph.D. thesis, Massachusetts Institute of Technology, Cambridge, MA, USA, 1990.

[9] J. N. Newman, "Distributions of sources and normal dipoles over a quadrilateral panel," Journal of Engineering Mathematics, vol. 20, no. 2, pp. 113-126, 1986.

[10] X. Chen, R.-c. Zhu, J. Zhao, W.-j. Zhou, and J. Fan, "Study on weakly nonlinear motions of ship advancing in waves and influences of steady ship wave," Ocean Engineering, vol. 150, pp. 243-257, 2018.

[11] W. Sun, H. L. Ren, H. Li et al., "Numerical solution for ship with forward speed based on transient Green function method," Journal of Ship Mechanics, vol. 18, no. 12, pp. 1444-1452, 2014.

[12] A. H. Clément, "An ordinary differential equation for the Green function of time-domain free-surface hydrodynamics," Journal of Engineering Mathematics, vol. 33, no. 2, pp. 201217, 1998.

[13] Z.-F. Li, H.-L. Ren, X.-W. Tong, and H. Li, "A precise computation method of transient free surface Green function," Ocean Engineering, vol. 105, pp. 318-326, 2015.

[14] W. Sun and H. L. Ren, "Ship motions with forward speed by time-domain Green function method," Chinese Journal of Hydrodynamics, vol. 33, no. 2, pp. 216-222, 2018.

[15] R. Datta, J. M. Rodrigues, and C. Guedes Soares, "Study of the motions of fishing vessels by a time domain panel method," Ocean Engineering, vol. 38, no. 5-6, pp. 782-792, 2011.

[16] H. Yasukwa, "Simulations of ship maneuvering in waves: 1st report: turning motion," Journal of the Japan Society of Naval Architects and Ocean Engineers, vol. 4, pp. 127-136, 2006.

[17] H. Yasukwa, "Simulations of ship maneuvering in waves: 2nd report: zig-zag and stopping maneuvers," Journal of the Japan Society of Naval Architects and Ocean Engineers, vol. 7, pp. 163-170, 2008.

[18] J. Zhu, Y. J. Pang, and Y. R. Xu, "Maneuvering prediction of a ship in regular waves," Journal of Harbin Engineering University, vol. 25, no. 1, pp. 1-5, 2004.

[19] X. F. Zhang and Y. Yin, "Ship motion mathematical model with six degrees of freedom in regular wave," Journal of Traffic and Transportation Engineering, vol. 7, no. 3, pp. 40-43, 2007.
[20] W. Zhang, Z.-J. Zou, and D.-H. Deng, "A study on prediction of ship maneuvering in regular waves," Ocean Engineering, vol. 137, pp. 367-381, 2017.

[21] J. Wang, L. Zou, and D. Wan, "CFD simulations of free running ship under course keeping control," Ocean Engineering, vol. 141, pp. 450-464, 2017.

[22] R. Subramanian and R. F. Beck, "A time-domain strip theory approach to maneuvering in a seaway," Ocean Engineering, vol. 104, pp. 107-118, 2015.

[23] T. I. Fossen, Guidance and Control of Ocean Vehicles, John Wiley \& Sons, Hoboken, NJ, USA, 1994.

[24] W. W. Bai, Identification modeling for ship maneuvering motion based on locally weighted learning, Ph.D. thesis, Dalian Maritime University, Dalian, China, 2018.

[25] P. C. Westlake and P. A. Wilson, "New conformal mapping technique for ship sections," International Shipbuilding Progress, vol. 47, pp. 5-22, 2000.

[26] J. M. J. Journée, “Theoretical manual of SEAWAY," Report 1216a, Delft University of Technology, Delft, Netherlands, 2001.

[27] T. Zhang, J. S. Ren, X. Q. Fan et al., "Simulation on ship heave and pitch motion based on multi-parameter conformal mapping method," Journal of Shanghai Maritime University, vol. 40, no. 3, pp. 51-56, 2019.

[28] X. Zhang, P. Bandyk, and R. F. Beck, "Seakeeping computations using double-body basis flows," Applied Ocean Research, vol. 32, no. 4, pp. 471-482, 2010.

[29] X. G. Lu, "Tasai method and program development for fluid dynamic coefficient calculation of ships," Master thesis, Tianjin University, Tianjin, China, 2003. 